\title{
Commissioning of the ATLAS inner detector with cosmic rays
}

\section{M.J.Costa*}

Instituto de Física Corpuscular IFIC CSIC-UVEG

E-mail: Maria.Jose.Costa@ific.uv.es

\section{C.Schmitt}

CERN

E-mail: Christian.Schmitt@cern.ch

On behalf of the Inner Detector software commissioning group.

The inner detector of the ATLAS experiment is in the process of being commissioned using cosmic ray events. First tests were performed in the SR1 assembly hall at CERN with both barrel and endcaps for all different detector technologies (pixels and microstrips silicon detectors as well as straw tubes with additional transition radiation detection). Integration with the rest of the ATLAS sub-detectors is now being done in the ATLAS cavern.

The full software chain has been set up in order to reconstruct and analyse this kind of events. Final detector decoders have been developed, different pattern recognition algorithms and track fitters have been validated as well as the various alignment and calibration methods. The infrastructure to deal with conditions data coming from the data acquisition, detector control system and calibration runs has been put in place, allowing also to apply alignment and calibration constants. The software has also been essential to monitor the detector performance during data taking. Detector efficiencies, noise occupancies and resolutions have been studied in detail and compared with those obtained from simulation.

XI International Workshop on Advanced Computing and Analysis Techniques in Physics Research April 23-27 2007

Amsterdam, the Netherlands

\footnotetext{
* Speaker.
} 


\section{Introduction}

The LHC designed luminosity of $10^{34} \mathrm{~cm}^{-2} \mathrm{~s}^{-1}$ implies a big challenge for the design of the ATLAS inner detector which needs to cope with high instantaneous and integrated rates: fast detector and readout electronics are required to read data at $40 \mathrm{MHz}$, high granularity should be achieved to deal with around $10^{3}$ charged particles and all devices should be radiation hard to keep operating after 10 years of LHC operation.

The ATLAS inner detector combines three different detector technologies: $50 \times 400 \mu \mathrm{m}$ Pixels in the inner layers, microstrip silicon detectors (SCT) in the intermediate radial region (with $80 \mu \mathrm{m}$ pitch in the barrel and 57-90 $\mu \mathrm{m}$ pitch in the endcaps) and $4 \mathrm{~mm}$ diameter straw tubes with additional transition radiation detection (TRT) in the outer part. However, during the commissioning period of the TRT, an Argon based gas mixture is used instead of a Xenon based gas and therefore only the tracking capabilities of the TRT can be studied.

The barrel is made of 3 layers of Pixels, 4 SCT layers and 73 straw layers and each endcap contains 3 Pixel disks, 9 SCT disks and 14 TRT wheels, having a total length of $5.7 \mathrm{~m}$ and a radius of $115 \mathrm{~cm}$.

In order to get ready for the first LHC collisions the commissioning of the inner detector is divided in two different phases.

- Phase 1: Since most of the detector integration is performed above ground in the SR1 assembling experimental site, it has been considered essential to test the different assembled structures (SCT-TRT barrel and endcaps and Pixel barrel and endcaps) before installation in the ATLAS cavern by performing full calibration runs to check for noise and in some cases by collecting cosmic rays in order to study its tracking performance. Cosmic data has been recorded so far by part of the SCT-TRT barrel (1/4 of the SCT and 1/8 of the TRT), part of one of the SCT-TRT endcaps (1/4 of the SCT and 1/16 of the TRT) and one Pixel endcap. An external trigger given by a set of scintillators was used. During the endcap test also an alternative trigger strategy based on coincidences between different wheels of the TRT endcap was used.

- Phase 2: After installation in the ATLAS cavern, the aim is to take cosmic rays together with the other ATLAS detectors as they are being installed. First data with $1 / 6$ of the TRT barrel has already been collected but unfortunately not enough to provide calibration and alignment constants. The SCT has also been integrated in the combined acquisition but sending noise corresponding to four SCT module prototypes. In these combined ATLAS runs, an internal ATLAS trigger is used, given either by the muon trigger chambers of by the hadronic calorimeter and no magnetic field was available.

\section{Reconstruction and simulation software}

The complete reconstruction chain has been put in place in order to deal with both cosmic events and real data. The same chain can also be applied to simulated data, but for real data an additional step is required. This additional step consists on decoding the data given by the different sub-detector readout drivers (RODs) which is in byte stream format and provide the raw 


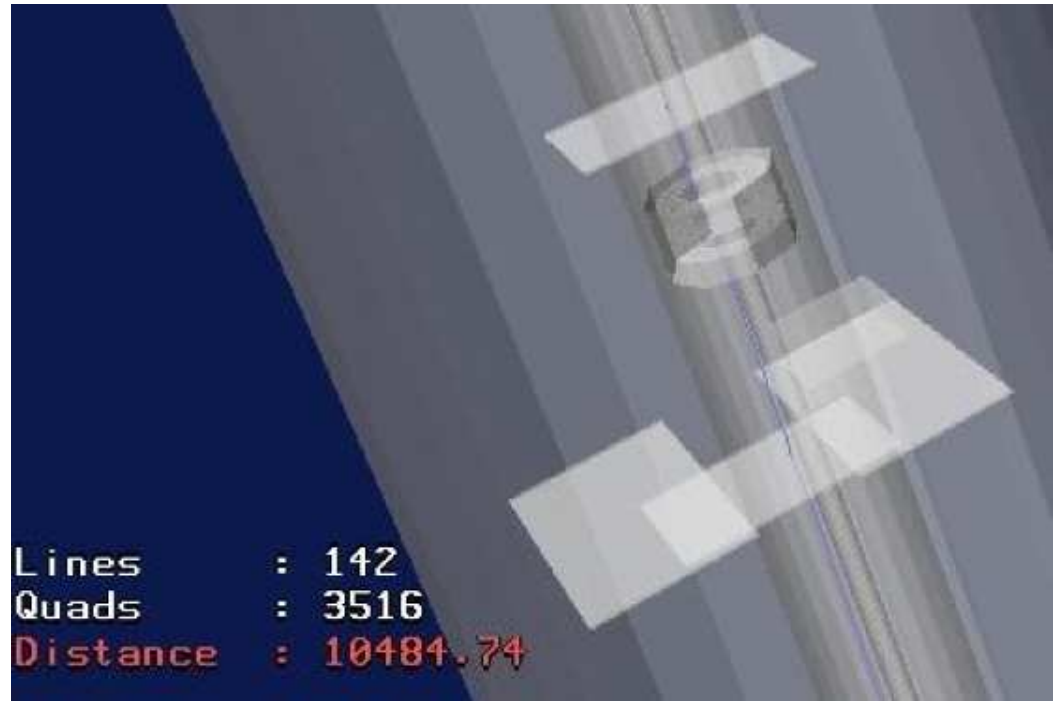

Figure 1: Visualization of the detector description used to simulate and reconstruct the SR1 endcap A Pixel.

data objects, as input to the reconstruction. These decoders need to access information from the conditions database to get the cabling maps (i.e. which detector element is connected to which link of the ROD).

The raw data objects are then translated into positions in space producing the so-called silicon clusters, space points ${ }^{1}$ and TRT drift circles. This step requires the implementation of a detector description of the corresponding setup in order to obtain the geometry information (including alignment corrections retrieved from the conditions database as well). As an example, Figure 1 shows the Geant 4 [1] visualization of the Pixel setup. This process requires additional access to the conditions database in order to mask those channels identified as bad by either the data acquisition, detector control system, calibration or monitoring algorithms, and to obtain the TRT R-t calibrations.

The resulted calibrated objects are then the input to the pattern recognition algorithms and fitting tools to finally get the list of reconstructed tracks per event. Two different track fitters are used: global $\chi^{2}$ minimization [2] or a Kalman Filter [3] technique.

Furthermore, cosmic events have some particularities with respect to LHC collisions. They are not synchronized with the detector readout clock since they occur randomly. That has to be taken into account in the TRT calibration method and requires a specific readout for the SCT in order to maintain high efficiency. The other obvious difference is that they do not come from the ATLAS center or from a given point in space, so the pattern recognition and SCT space point creation algorithms cannot use any vertex constrain.

A simulation of the different cosmic setups have also been provided to first set up and validate the full reconstruction chain and then to allow for data/Monte Carlo comparisons which could lead

\footnotetext{
${ }^{1}$ The SCT modules are made of two silicon planes with a rotation of $40 \mathrm{mrad}$ among them. The clusters contain the measurements provided by a single plane while the space point combined the information of the two planes by assuming a particle direction.
} 


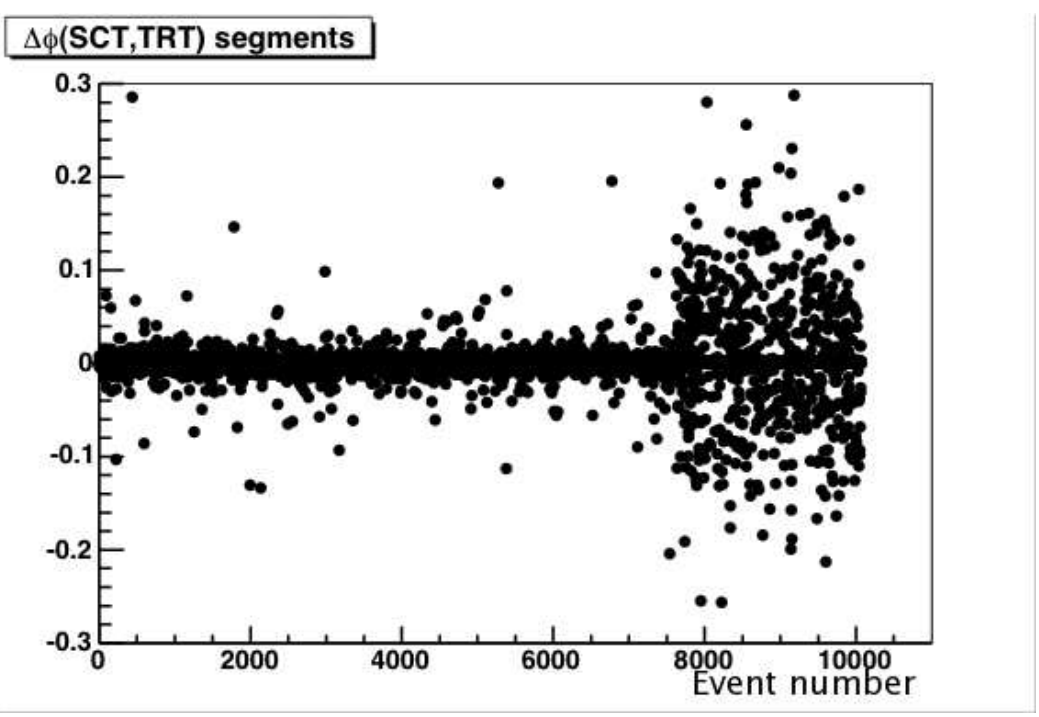

Figure 2: Difference between the SCT and TRT reconstructed track $\phi$ parameter as a function of the event number for the combined barrel test.

to improvements on the current simulation.

\section{Monitoring}

The full reconstruction chain can also run online at the third trigger level to allow for an online monitoring that continuously checks the good data quality and detector performance before events are written to disc. Different monitoring tools have then be developed in order to produce histograms out of the different reconstructed objects (raw data information, silicon clusters and space points, TRT drift circles and the final tracks). The updated histograms are then periodically sent to the Online Histogram Server (OHS) and are collected by an interactive GUI called the Online Histogram Presenter (OHP) with a frequency set by the user [4].

Figures 2 and 3 shows an example of two of the histograms displayed online during the SR1 combined cosmic run performed with the SCT and TRT barrel. The former checks for the SCT and TRT synchronization by looking at the difference between the SCT and TRT barrel $\phi$ track parameter as a function of the event number. It can clearly be observed that synchronization is lost after around 7000 events.

The histogram of Figure 3 shows the variation of the noise occupancy on the TRT endcap C with the event number. In this case, an increase of the noise occupancy from around $2 \%$ to $6 \%$ due to a tripp of the analog low voltage regulators was detected.

In addition to the produced monitoring histograms, it was also possible to run the ATLAS event displayed (Atlantis) online. Figure 4 shows two examples of event displays taken during the combined SCT and TRT barrel (left) and endcap (right) cosmic runs. Figure 5 shows an example of an event collected in the ATLAS cavern by the TRT and the calorimeters and muon chambers.

During the combined ATLAS data taken in the ATLAS cavern, for the first time the offline monitoring at the Tier-0 [5] was exercised. The full ATLAS combined reconstruction chain was 


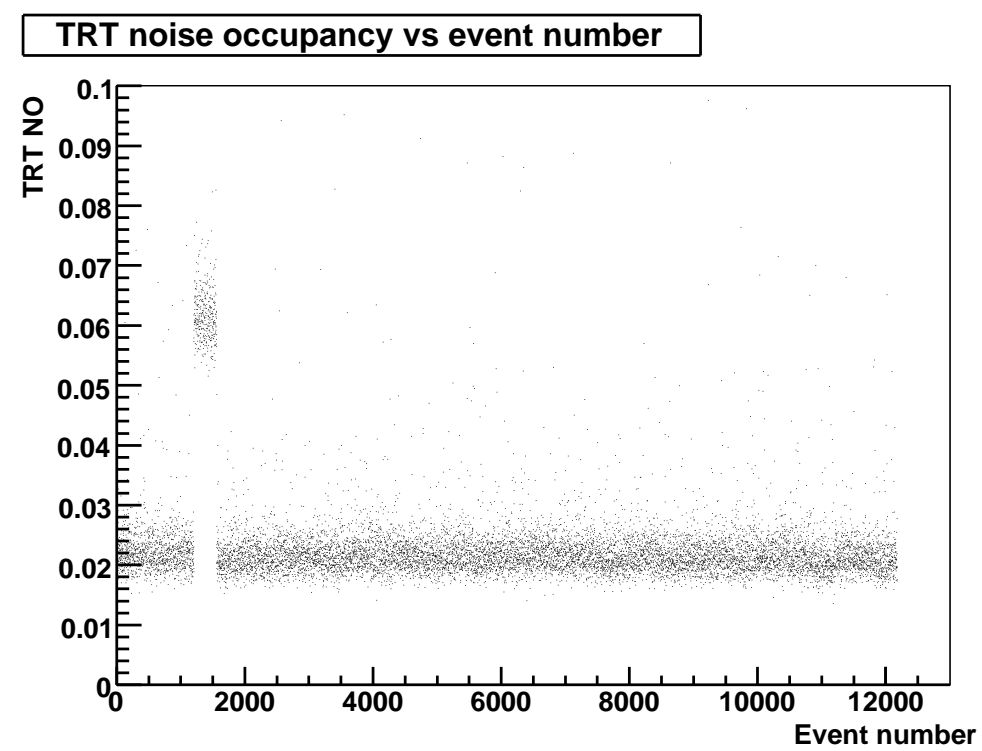

Figure 3: TRT endcap C noise occupancy as a function of the event number.
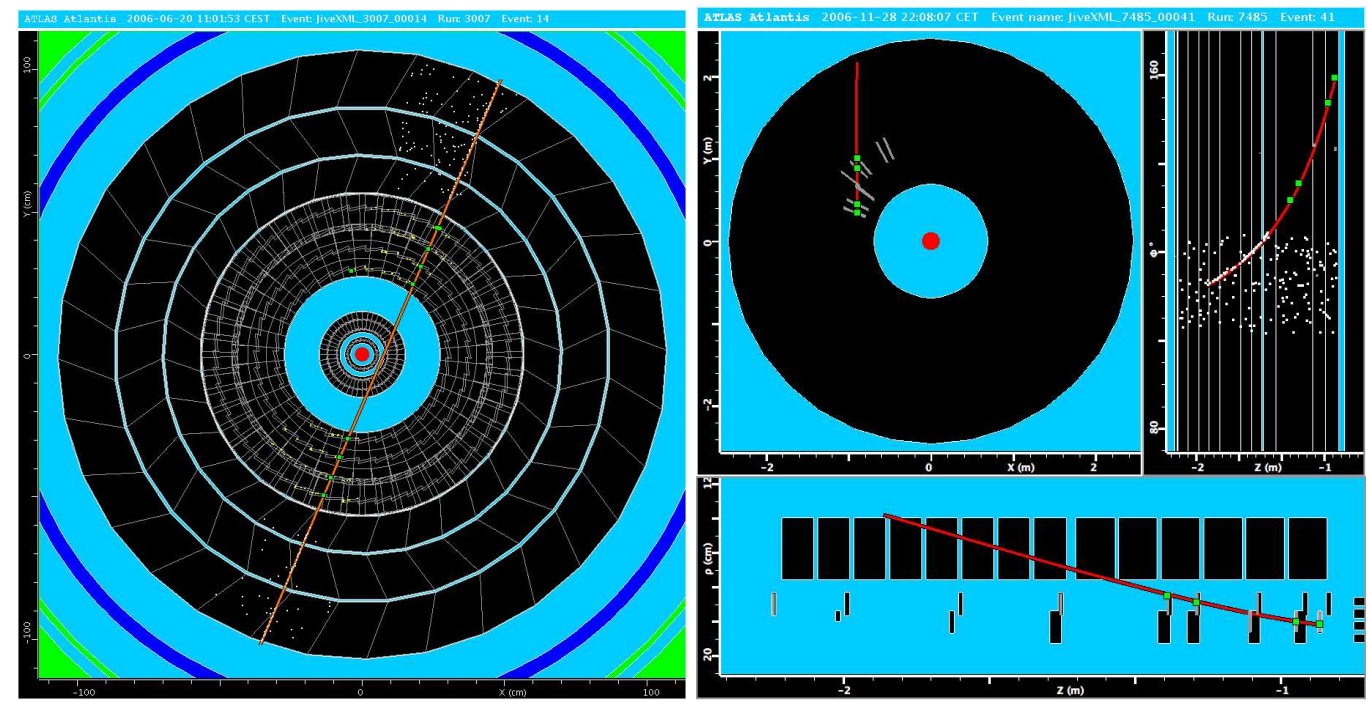

Figure 4: An Atlantis event display during the combined SCT and TRT barrel (left) and endcap (right) cosmic run.

continuously running as the data was becoming available producing as output monitoring histograms which were sent to the data quality webpage accessible from the control room as well as the different input required by the analysis algorithms. 


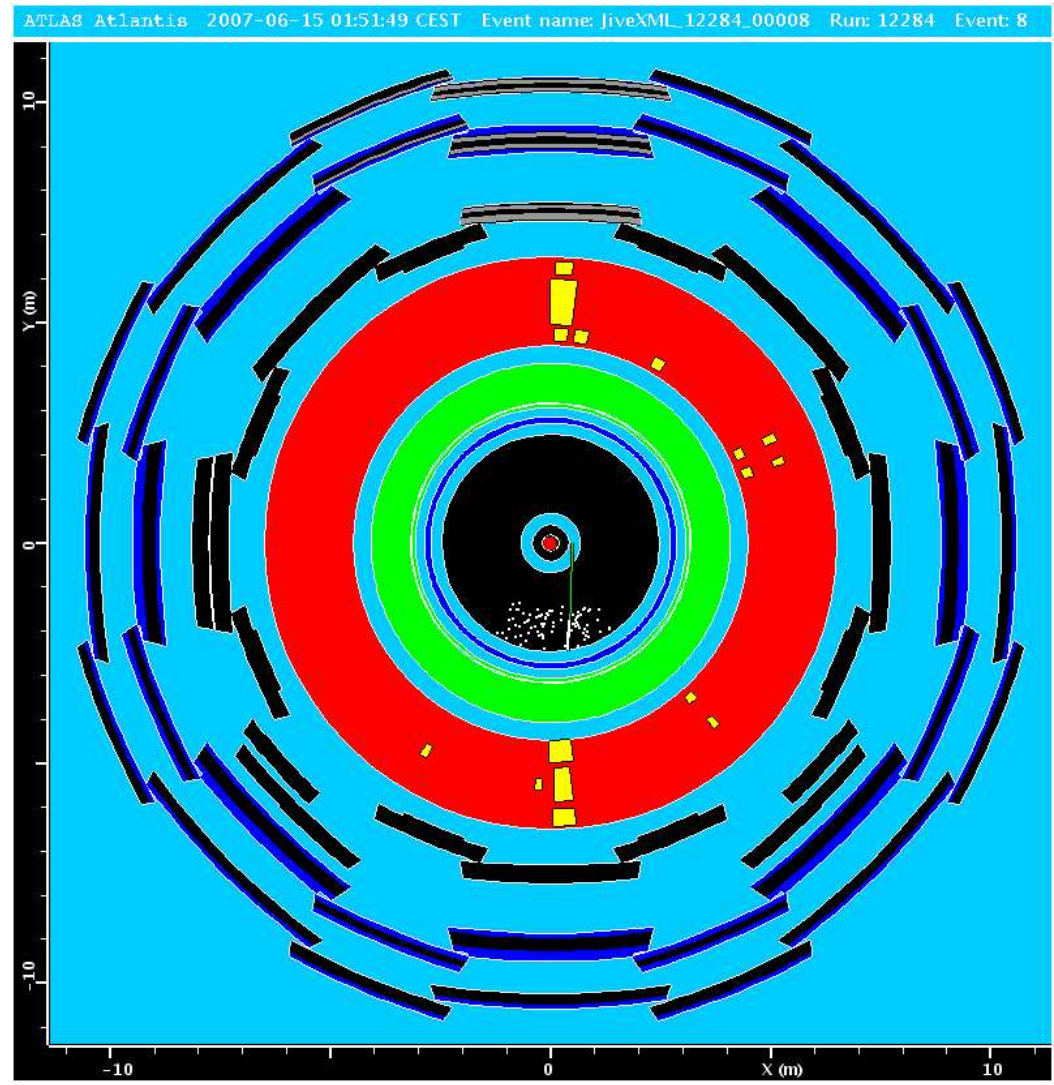

Figure 5: An Atlantis event display during the combined ATLAS run in the cavern.

\section{Data analysis results}

\subsection{Tests with random triggers}

The noise on the different sub-detectors (Pixels, SCT and TRT) was tested for many different configurations by analysing the data collected in runs with random triggers.

The Pixel detector is tuned for a threshold of $4000 \mathrm{e}$, to which corresponds a noise of about 120 e. The observed noise occupancy was $1-2 \times 10^{-8}$ per bunch crossing. This is mostly due to the fixed pattern noise caused by a fraction $10^{-3}$ channels with an occupancy of $10^{-5}$. Comparison with the detector characterization performed during the detector assembly shows that most of these channels were already detected by tests with a 241 Am source (see Figure 6).

Concerning SCT, the distribution of the measured noise occupancies for all active module sides of the barrel and endcap C (outer and middle) are shown in Figure 7 which has a mean of $4.76 \times 10^{-5}$. Concerning the inner endcap modules, statistics at $1 \mathrm{fC}$ threshold is too small to extract a reliable value. All numbers are within specifications.

In addition, the noise was tested for several different conditions: varying the trigger rate from $5 \mathrm{~Hz}$ to $50 \mathrm{KHz}$, having the TRT on and off, with the heaters on, off and switching between the two states, several grounding schemes and while the TRT was being readout. No increase of noise was observed in any of the tested configurations. 


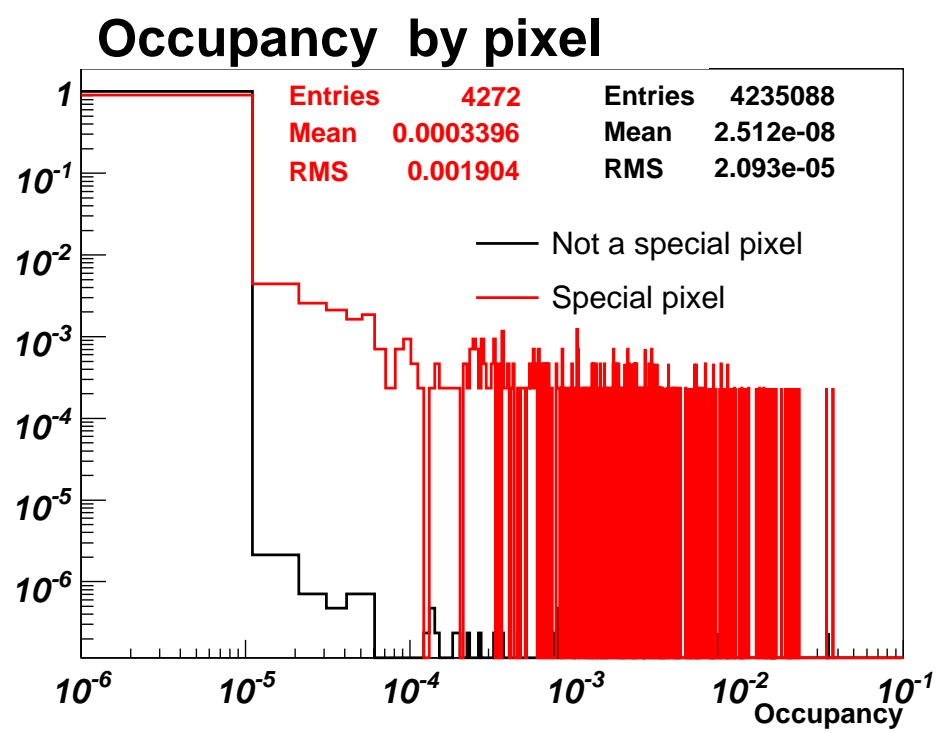

Figure 6: Distribution of the pixel noise occupancy measured in SR1 over 16 bunch crossings. The first bin contains as well the lower occupancy pixels.

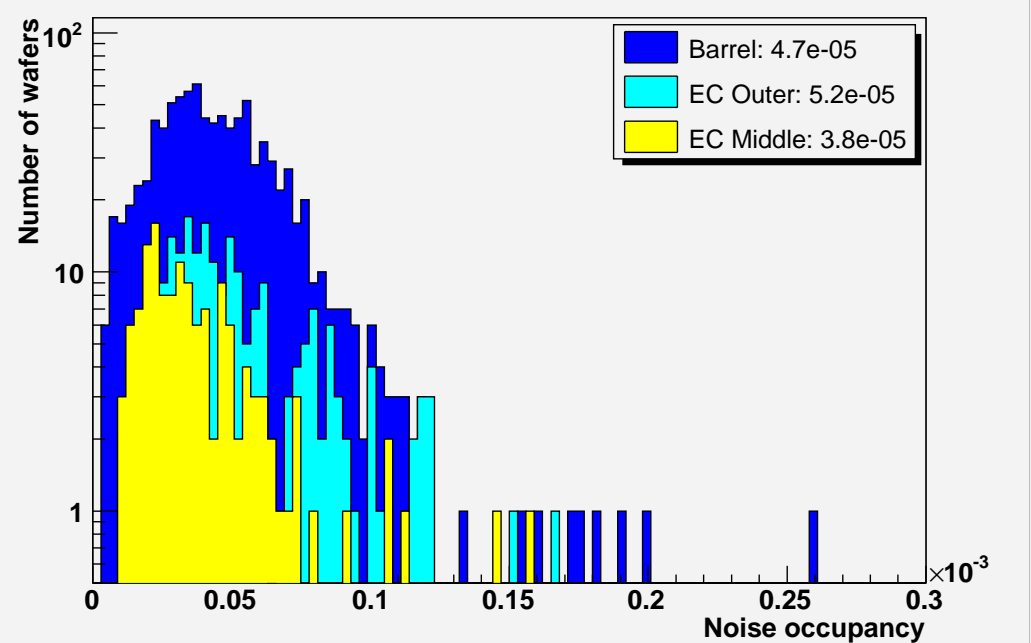

Figure 7: (Distribution of noise occupancies for all active module sides of the SCT barrel and endcap obtained at $1 \mathrm{fC}$ threshold. 
Similar tests were also performed for the TRT barrel: the noise was verified before and after the insertion of the SCT, in a configuration in which the TRT analog ground was connected to the SCT power return, for different SCT thresholds and while the SCT was being readout. The straw noise occupancy was in all cases around $2 \%$.

\subsection{Tests with cosmic rays triggers}

The most relevant results that can be obtained from cosmic rays data are the detector resolutions and efficiencies. In order to do that it is essential to first align and calibrate the detector and for that the different available methods designed [6] to align the system using LHC data have been used for the SCT and TRT. Concerning the Pixels, in the setup used at SR1, pixel detector only tracks have little redundancy and it is difficult to implement a full detector alignment.

Due to the lack of magnetic field in the data taken so far, material effects could not be taken into account in the tracking algorithms and therefore track uncertainties are not properly estimated. It is then not trivial to obtain a good measurement of the detector resolution since that comes from the standard deviation of the residual distribution after subtracting the track uncertainty. The approach followed in order to get such an estimate was to look at the $\sigma$ of the residual distribution as a function of the unbiased $\chi^{2}$ for each given hit (i.e. the $\chi^{2}$ of the track removing the contribution of the hit under evaluation). This dependence is shown in figure 8 for the TRT barrel and a $\sigma$ of about $170 \mu \mathrm{m}$ is obtained when $\chi^{2}$ tends to 0 , consistent with what it is expected for the Ar based gas mixture used in the commissioning phase of the TRT.

Efficiencies have also been computed for each sub-detector, being all of them within specifications (see Figure 9 and 10 for the SCT and TRT barrel respectively).

\section{Conclusions}

The ATLAS inner detector is being commissioned with cosmic rays taken first above ground with the different assembled structures and then underground in the ATLAS cavern together with the calorimeters and muon chambers. The reconstruction, alignment, calibration and monitoring chains have been put in place to deal with real data and cosmic rays events and are providing prompt feedback to the detector performance. Results show that the detector is well within specifications in terms of noise, efficiency and resolution. A simulation of the different setups has also been provided to allow for a tuning of the Monte Carlo and to prepare the full chain before dealing with real data.

\section{Acknowledgments}

The author is indebted to the whole ATLAS inner detector commissioning group but would like to acknowledge specially A. Andreazza, T.Cornelissen, H. Hayward, W. Hulsbergen, A.C. Le Bihan, R. Wall, M. White, W. Yao, M. Zdrazil for their input to this note.

\section{References}

[1] S.Agostinelli et al., Nucl. Inst. and Meth. A (2003) 506; J.Allison et al., IEEE Transactions on Nuclear Science (2006) 53. 


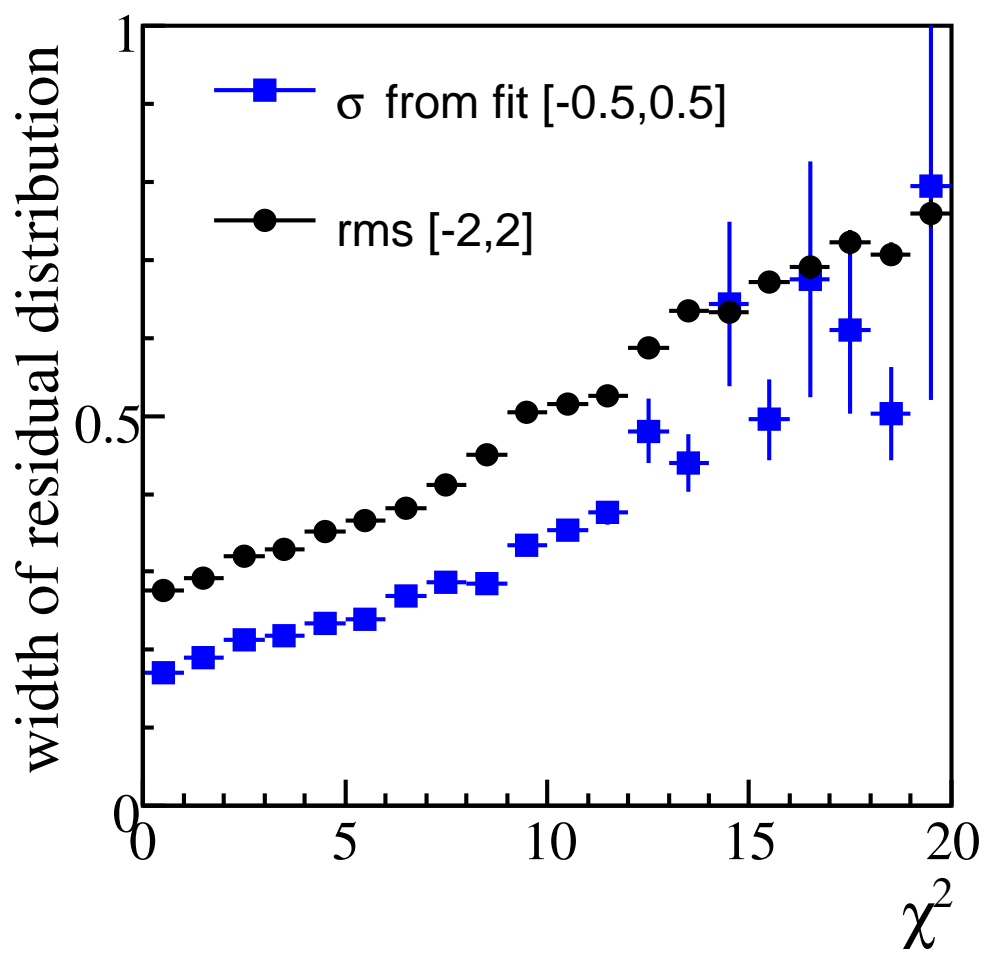

Figure 8: (Left) rms and $\sigma$ of the TRT barrel residuals as a function of the $\chi^{2}$ of the track.

[2] T.Cornelissen, ATLAS note ATL-COM-INDET-2006-003.

[3] R.Frühwirth, Nucl. Inst. and Meth. A262 (1987) 446.

[4] A.Dotti, proceedings of Computing in High Energy and Nuclear Physics 2006.

[5] L.Goossens et al., proceedings of Computing in High Energy and Nuclear Physics 2006.

[6] Ameling et al., CERN yellow report, CERN-2007-004 


\section{Efficiency per layer}

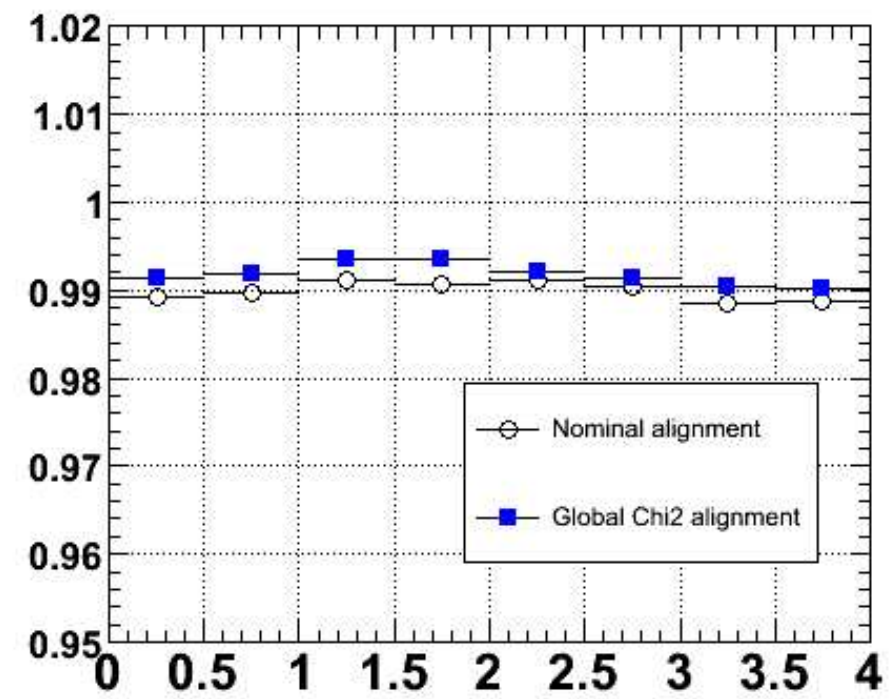

Figure 9: SCT efficiency as a function of the layer side for the case in which no alignment corrections have and have not been applied.

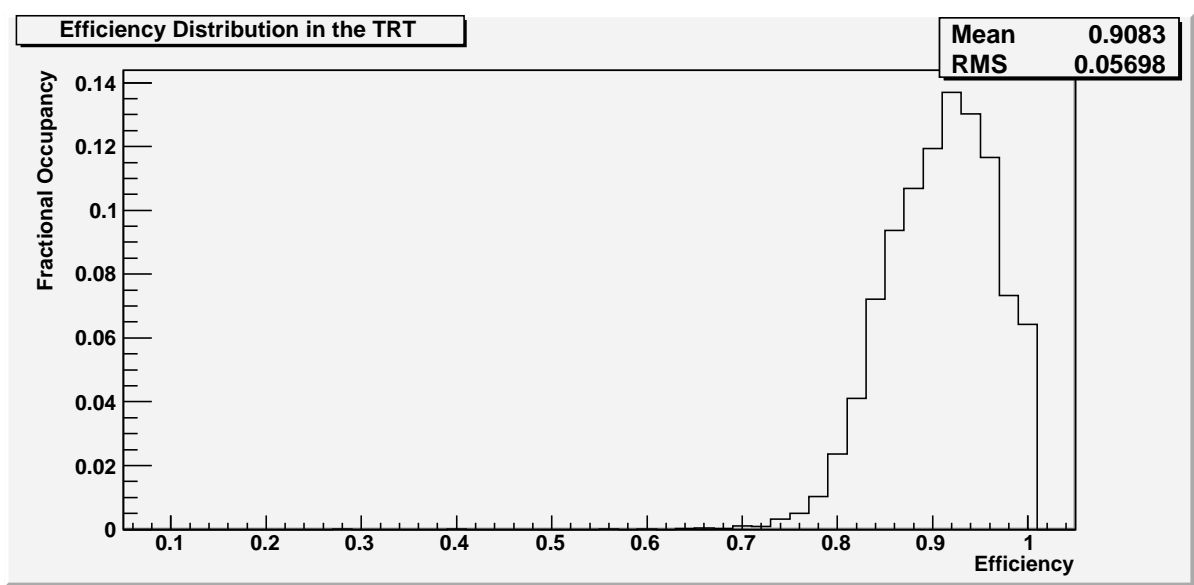

Figure 10: Distribution of the TRT barrel straw efficiencies. 